\title{
Updates for Authors and Reviewers
}

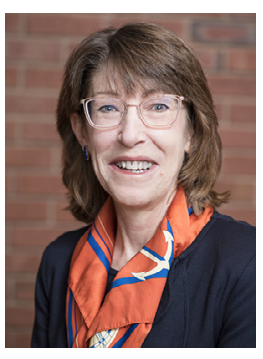

If you're writing a manuscript, planning a project, or agreeing to review for JNEB, do you read the Guidelines for Authors and Reviewers? They can be quite long. We mostly know what they say, right? An editor will correct things or a reviewer will suggest changes. Perhaps. However, we've been making some changes at JNEB that I wanted to share.

The most obvious change is we no longer prohibit the use of first-person pronouns. Hopefully, this will eliminate awkward phrasing and passive voice so that the paper is easier to write and read. No more "the researchers conducted..." and onto "we conducted..."!

Times change and so does the publishing world. Have you heard of a preprint or published a preprint? Preprints are manuscripts that are shared by authors to invite comments from the research community and to establish that innovations have been made by the researchers. Authors may post their work on preprint servers and websites; however, these papers have not been through the peer-review process. But they are "published," and so submitting them to JNEB for publication again can be problematic. There are many types of servers that "publish" manuscripts, and each has its own licensing or copyright agreement. Read the fine print before publishing a preprint. If there is no copyright transfer from the authors and no license agreement, JNEB may consider the manuscript. However, if the manuscript is accepted, the authors are responsible for linking the preprint to the published paper. If that is not possible, JNEB must decline consideration of the manuscript. We plan to have a webinar to discuss preprints and the publishing world soon.

Statistical analyses keep all of us on our toes. A couple of changes at JNEB related to statistics is our screening of cluster randomized control trials. Upon submission, these manuscripts will be screened to be sure that:

- The number within a group, the number of groups, and the strength of group-level dependency (intraclass correlation coefficient [ICC]) are to be considered.

- A power analysis using the outcome variable of interest and in consideration of the cluster is addressed.

- The model type is included: fixed effects, random effects, or mixed effects with consideration of the cluster and a reference or explanation as to why this model was chosen.

Finally, for intervention studies, a power analysis should be included with the effect size, alpha, power, outcome variable used, the analysis considered (ANCOVA, $t$ tests, etc), software used for the calculation, and the number of participants the power analysis indicated. For pilot intervention studies, if the pilot study focuses on the adaptability and/or feasibility of an innovative intervention, a power statement is optional. However, it should be obvious from the title, abstract, objective, and narrative that this is a pilot. In the abstracts of intervention studies, pilot or not, nonsignificant results in interventions should be reported only for those variables that were powered to see an effect.

All of us at JNEB work very hard to stay current with all aspects of nutrition education and behavior research, ethics, and guidelines. One area that is always in our discussions is diversity, equality, and inclusiveness. At a very basic level, JNEB is more attuned to data of sex and gender and how these data are collected. From our Guidelines for Authors:

JNEB supports gender neutrality by using plural nouns (clinicians, educators, participants) as default wherever possible and avoid using "he, she," or "he/she" but rather "they." According to the AMA, "sex refers to the biological characteristics of males and females. Gender includes more than sex and serves as a cultural indicator of a person's personal and social identity." Recognition of the diversity within gender self-identity is important for researchers and practitioners in nutrition education and behavior. Methodology for collecting data related to gender and sex should be transparent within the Methods section, including but not limited to data that are: self-reported in an open-ended response option, or a choice format that allows for multiple selections, a single selection, or no response. A rationale for data analyses for sex or gender should be clear in the study design and objectives. If sex and gender data are collected only for the description of the sample and are not part of the design, this should also be clear in the results.

As we continue to think and talk about advances and changes in research and publishing, more guidelines will be updated. As always, if you have concerns or thoughts, please contact me at editor@jneb.org.

With kind regards, Karen Chapman-Novakofski $P h D, R D N$ Editor-in-Chief 